\title{
Meter of Initial Speed of Mobile Object
}

\author{
A.P. Kozlov \\ National Aviation University \\ Kyiv, Ukraine \\ ap_kozlov@ukr.net
}

\author{
A.S. Yurchenko \\ National Aviation University \\ Kyiv, Ukraine \\ ayurchenko@yahoo.com
}

\begin{abstract}
The need to measure the initial velocity of a moving object is shown. A brief review and analysis of existing technical means suitable for solving the problem is given. The constructions of devices and their elements for measuring the initial velocity of a mobile object, structural diagrams of devices for measuring the initial velocity of a mobile object are considered. A design with a capacitive converter is proposed, which forms an information impulse. The results of the development can be used to design and manufacture the device.
\end{abstract}

Keywords-initial velocity; mobile object; geodetic method; capacitive converter; information impulse

\section{INTRODUCTION}

The state of the economy of Ukraine today requires more extensive automation of production in various areas of domestic industry, the introduction of new automation tools and their further improvement. First of all, this refers to serial production. Automation of the technological process of manufacturing parts, assembly of product units makes it possible to create competitive products. Means of automation, robotics can increase the volume of products, reduce the cost of production, reduce the cost and price of products, while ensuring its high quality. In turn, this increases the demand for products and profitability of production. The development and implementation of automation tools in the domestic industry are relevant. To automate the technological process of manufacturing or assembling a technical object or its parts, it is necessary to perform measurements of its movement parameters: displacement, speed, acceleration, angular displacements, etc. To measure these parameters, it is necessary to have devices with which a measurement with the required accuracy is possible. In particular, it is often necessary to measure the initial exit speed of a part from a manufacturing device in order to calculate the movement parameters of the gripping device, which ensures that the part is transferred to the next processing unit. Proceeding from the above, it is obviously necessary to develop a measuring instrument for the initial velocity of a mobile object.

\section{ANALYSIS OF PROBLEM}

To measure the initial velocity of a mobile object, a device is required that satisfies the conditions described above. To do this you need:

1) to review and analyze existing methods and technical means in order to find suitable technical solutions;

2) to develop the design of a measuring instrument that implements the chosen method;
3) develop a structural and functional scheme of the meter.

The review and analysis of existing methods and technical means of measuring the speed of movement of a mobile object showed the following. To measure the speed of mobile objects, aerometric, thermal, thermodynamic, mechanical, locational, Doppler, inertial, geodetic methods of measurement are used. Of the methods listed, only locational and geodetic methods for measuring the speed of a mobile object can be considered, since the implementation of devices constructed using these methods requires the integration of a meter into the mobile object, which is unacceptable in this case.

The locational method of measurement, depending on the frequency of oscillations of the air or electromagnetic field, is divided into ultrasonic (acoustic), radio engineering, optical and laser methods [1]-[3]. Basically, the location method is used to measure distances, distances to a mobile object, its movements. To evaluate the speed of the movement of the object, it is necessary to differentiate the information signal. This, in turn, leads to additional errors, which are caused by differentiation of the noise component of the useful signal, which often leads to the inexpediency of applying the method.

In order to measure the initial velocity of a mobile object, the geodetic method is, in our opinion, the most acceptable one.

The essence of the geodesic method is as follows: the time interval $\Delta \mathrm{t}$ is measured, for which the mobile object passes some basic distance. The basic distance may be the longitudinal dimension of the movable object, or the distance between the reference points of a specially designed structure. If the longitudinal dimension of the mobile object $\mathbf{L}$ is taken as the reference distance, then the object's speed is defined as $\mathbf{V}=\mathbf{L} / \boldsymbol{\Delta t}$, where $\Delta \mathbf{t}$ is the time interval for which the mobile object passes the reference distance.

To form an information pulse, inductive, photometric or capacitive sensors (converters) are used. The information impulse is obtained by including the sensor in any measuring circuit, for example, the scheme of an AC measuring bridge, at the output of which an information pulse is formed.

The inductive sensor is a round coil, the inductance of which changes as the mobile object moves through the coil. Measurement of the pulse duration, as a rule, is realized by digital microcircuits. It should be noted that for the efficient operation of the induction sensor, it is necessary that the mobile object has magnetic properties, i.e., it had, for 
example, a steel non-magnetized body. On a non-magnetic material (brass, bronze, plastic, etc.), the sensor will not respond.

The photometric method for obtaining the information pulse is based on the interruption of the light beam by the mobile object. A sensor built on this principle is a system of "light source - a light detector", usually an "LED-photodiode". To increase the reliability of obtaining useful information, the sensor has a special design on which several radiators and photodetectors are placed. Two sensors located at the base distance $\mathbf{L}$ allow to measure the initial speed of the mobile object according to the above algorithm. This method is subject to external influencing factors, such as external radiation, dust and air pollution, which reduces the effectiveness of its use.

More acceptable, in our opinion, is the formation of an information impulse with the help of capacitive sensors. To measure movement in engineering capacitive sensors are widely used. The overwhelming majority of technical solutions use sensor designs with a homogeneous field (Fig. 1).

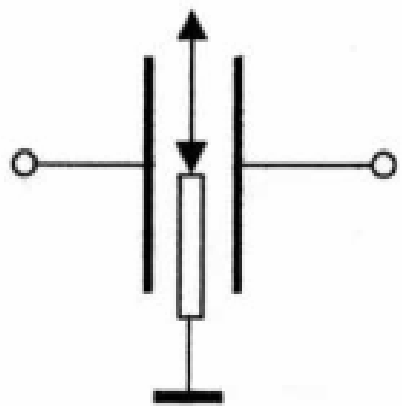

Fig. 1. Design of the sensor with a homogeneous field.

In order to eliminate the influence of the edge (inhomogeneous) field in modern designs, screens and socalled guard electrodes are used [4], [5]. As can be seen from Fig. 1, the moving object must be grounded and the sensor electrodes must be insulated. The length of the mobile object is taken as the base distance $\mathbf{L}$. The formation of the information pulse is performed by including the sensor in the circuit of the four-arm ac bridge. The bridge is pre-balanced, When entering the interelectrode space of the mobile object, the balance is violated. The design of the device has small dimensions, determined mainly by the dimensions of the mobile object. The parameters of the air and gas environment practically do not affect the useful signal. However, the considered design and measurement scheme have a number of drawbacks. To form a uniform electromagnetic field, the transducer electrodes have a planar rectangular shape and are placed parallel to each other. To ensure the grounding of the mobile object, it is necessary to develop a special design. When a mobile object is placed in the interelectrode space, which has in general various forms, the uniformity of the field is violated. This affects the shape of the information pulse, but its duration, which determines the speed of motion, does not affect the inhomogeneity of the field. Thus, the possibility of using a capacitive transducer with an inhomogeneous field is obvious. In some sensors, for example in thickness gauges, because of the need to place the sensor on only one side of the material being monitored, sensor designs with a non-uniform ("open") field are used [6]. The static characteristic of the sensor in this case is nonlinear. The action of such sensors is based on the effect of the edge field (Fig. 2). The change in the capacitance of the sensor is caused by the introduction of a body whose dielectric constant differs from the dielectric constant of its surrounding medium, that is, in the space of the electromagnetic field created by the electrodes of the capacitive sensor. A sensor with an "open" inhomogeneous electromagnetic field is more suitable for solving the problem under consideration.

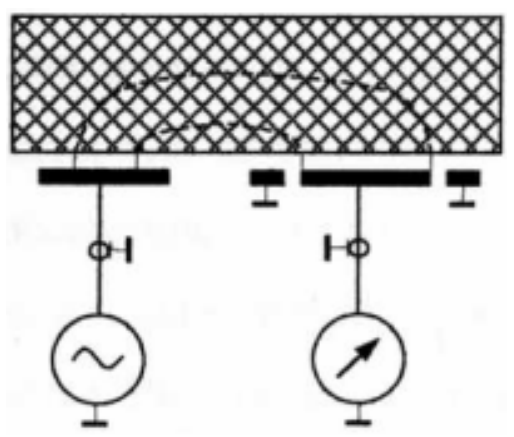

Fig. 2. Illustration of the effect of the edge field.

\section{Problem SOLUTION}

Taking into account the foregoing, we propose a scheme for constructing the device, shown in Fig. 3.

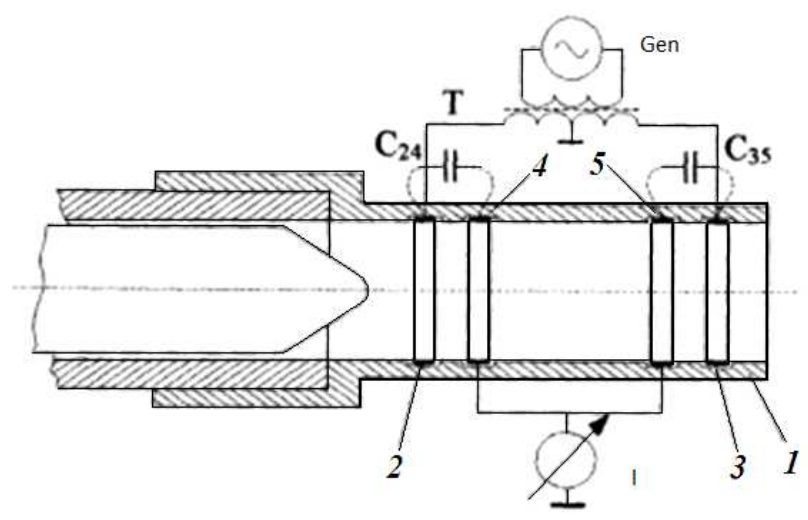

Fig. 3. To the principle of constructing an initial speed meter: 1 is the body of the speed measuring device; 2, 3 are high-potential electrodes; 4, 5 are lowpotential electrodes; $\mathrm{T}$ is the shoulder transformer; Gen is the power generator of measuring bridge; $\mathrm{I}$ is the indicator.

The body of the device 1 has internal sectional dimensions corresponding to the dimensions of the movable object. This can be a circular or rectangular section. On the inner surface of the body grooves are made, in which 4 sensors electrode $2-5$ are placed on insulators. Technically, the design is made folding. The housing of the device 1 is fixed to the output device. The electrodes 2, 4 and 3,5 form capacitors with an inhomogeneous field, which are included in the scheme of the transformer measuring bridge. In view of the identical design and dimensions of capacitor electrodes in the absence of a movable object, the measuring bridge will be balanced, that is, at the bridge output, the signal is practically zero. When the 
movable object overlaps with the first pair of electrodes 2,4 , the balance is violated, the signal appears at the bridge output (Fig. 4), the value of which rapidly increases as the mobile object covers the range of the capacitor $\boldsymbol{C}_{\mathbf{2 4}}$. At the subsequent movement of the mobile object, the zone of action of the capacitor $\boldsymbol{C}_{\mathbf{3 5}}$ is similarly overlapped. When the two objects overlap with the mobile object, the measuring bridge is balanced. The shape and duration of the first (starting) pulse are determined by the configuration of the front part of the mobile object and the distance between the zones of the capacitors. The analysis of the functioning of the first part of the device shows the possibility of determining the speed of motion along the duration of the first pulse, if the distance between the zones of capacitors $\boldsymbol{L}_{\boldsymbol{k}}$. is taken as the reference distance. Then the speed of motion is defined as $V=L_{k} / \Delta t_{1}$.

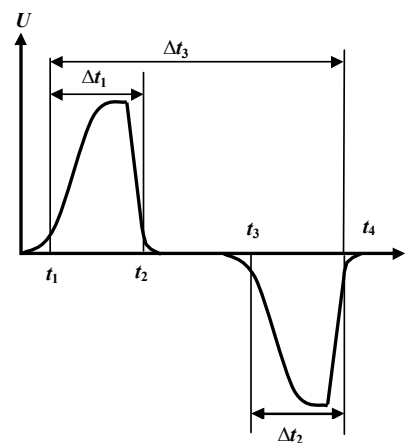

Fig. 4. Output signal of the capacitive converter of the initial speed meter.

The subsequent movement of the movable object opens the area of action of the capacitor $\mathrm{C}_{24}$. The bridge is unbalanced, but the unbalance signal will have the opposite sign. When the mobile object leaves the device, the bridge is balanced again. The received information impulse also gives information on the velocity $V=L_{k} / \Delta t_{2}$. If we use the longitudinal dimension of the mobile object $\boldsymbol{L}$ as the base distance, we can determine the velocity as $V=L / \Delta t_{3}$.

Thus, the capacitive converter outputs combined information, which allows to significantly improve the accuracy of the measurement when it is properly processed.

\section{RESUlTS}

To realize the considered principle of the device's action, a structural diagram of the speed meter has been developed (Fig. 5). The meter consists of a capacitive converter, which is a system of electrodes $\mathbf{S E}$, whose construction is shown in Fig. 3, shoulder elements ShE, which together with the system of SE electrodes constitute the measuring bridge, the preamplifier PA, the detector, the pulse shaper PS, the flipflop $\mathbf{T r}$, the clock pulse generator CPG, the logical element AND, the 10-bit counter and the calculator of value of initial speed $\mathbf{V}$, and also the power generator of the $\mathrm{AC}$ measuring bridge.

The scheme operates as follows. When the movable object overlaps with the action of the capacitor $\boldsymbol{C}_{\mathbf{2 4}}$, a signal appears at the bridge output. The signal is then detected and fed to the PS. Its use is necessary for reliable control of the Tr. The first pulse flips the trigger, the signal of which, entering the input of the AND circuit, opens the input through the second input of the logic circuit "AND" of the the clock pulse generator CPG to the counter input of the pulse counter. The second pulse flips the trigger, removing the enable signal. The account is terminated.

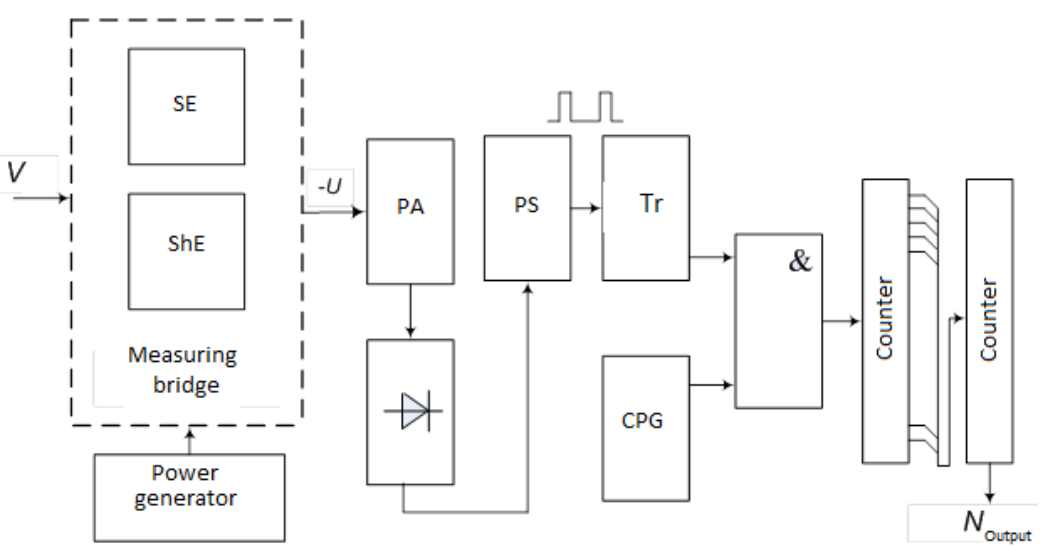

Fig. 5. Block diagram of the speed meter: SE is the system of electrodes; $\mathbf{S h E}$ are shoulder elements of the measuring bridge; $\mathbf{P A}$ is the preamplifier; PS is the pulse shaper; $\mathbf{T r}$ is the trigger; CPG is the clock pulse generator; \& is the logic diagram "AND".

\section{CONCLUSION}

The implementation of the system for detection of critical deformation elements of aircraft construction is proposed. The structure of sensitive element sensor is considered. The measuring system will ensure continuous monitoring of the state of the aircraft construction.

The structure of digital system for the detection of critical deformation elements of aircraft construction is considered.

\section{REFERENCES}

[1] V.L. Belsky, J. P. Vlasov, N.V. Zaitsev et al. Construction of aircraft. Moscow, Oborongiz, 1965, 710 p.

[2] S.A. Spector. Electrical measurements physical quantities, measurement methods. Leningrad, Energoatomisdat, 1987, 320 p.

[3] F.B. Grinevich and A.I. Novik. Test Compensatory and bridge Devices capacitive sensors. Kiev, Naukova Dumka. 1987, 112 p.

[4] A.K. Ablesimov, A.Z. Gaydamaka, and A.P. Kozlov. "Non-contact measurement of the micromovings." Elektroniki and the control system, Kyiv, NAU, vol. 3(5), 2005, pp. 141-146. 